\title{
Role of Dietary Pattern on the Microbial Diversity in the Gut of the Experimental Mice
}

\author{
Rosa J Samuel ${ }^{1}$, Omana $\mathrm{Joy}^{2}$, Prakash Piruthiviraj ${ }^{3}$ and Thamaraiselvi Kaliannan ${ }^{4}$ \\ ${ }^{1}$ Central Animal Facility, Indian Institute of Science, Bangalore, Karnataka, India \\ ${ }^{2}$ FACS Facility, Indian Institute of Science, Bangalore, Karnataka, India \\ ${ }^{1,3 \& 4}$ Laboratory of Molecular Bioremediation and Nanobiotechnology, Department of Environmental Biotechnology, \\ Bharathidasan University, Tiruchirappalli, Tamil Nadu, India \\ E-Mail: thamaraiselvi@gmail.com
}

\begin{abstract}
Animal models are important for biomedical research as they help us to understand the mechanisms and functions similar to humans. Microbiological analysis is an integral part of animal studies especially when looking for gut microbiota changes. Several factors such as diet, antibiotics etc. have an impact on the composition of the gut. While many conventional and molecular methods are used for the identification of microbiota, this study aimed at evaluating the usefulness of flow cytometry for identification of bacteria based on their ability to scatter light. Fresh gut samples from mice were collected and cultured on nutrient agar followed by plating on selective media. The isolates were characterized and differentiated by biochemical tests and FACS (fluorescent activated cell sorter) analysis. The major group of isolates obtained from culture methods and biochemical characterization were identified as Proteus and Klebsiella species. Forward and side scatter analysis of the samples showed two distinct patterns in the plots and correlated with the microbiological results. From the results it was found that flow cytometry can be effectively used as method of rapid detection of microorganisms from animal samples.
\end{abstract}

Keywords: Gut, Microbiota, Flow Cytometer, Rodents

\section{INTRODUCTION}

The use of animals for scientific and medical research is an age-old practice. This is due to the striking similarities found between humans and animals (especially mammals) which help researchers to study several mechanisms and novel therapeutic strategies in animal models before testing in humans. Humans and mammals are complex organisms with several physiological functions that occur in a highly regulated and integrated manner. Thus, study at multiple levels must be carried out in order to understand the various mechanisms and interactions. Animal models have been used for several scientific studies ranging from basic science to application of novel therapies, strategies or vaccines. Apart from being biologically similar, mammals are also affected by the same diseases found in humans. However, when using animals for biomedical research, care should be taken to check whether the animal is in good state of health to provide reliable and repeatable results. Some healthy animals are found to suffer from latent infections which often go unnoticed and symptoms may appear under stress during the experimentation. Infection, genetic, environmental and other factors must be considered for selection of suitable animal for research. Infectious agents were found to affect the expected results in areas such as physiology, oncology, immunology etc.

In India, scientific research using animal models is carried out in various subject areas namely cell biology, food technology, molecular biology, biomedical and biochemical research, pharmaceutical science, etc. Approximately one million animals are used per year for research purposes. Researchers are liable for maintaining the quality of animals used so as to produce reliable and reproducible data as well as follow ethical regulations for the use of animals for experimental purpose. Health monitoring must be an important part of the experimental plan and results should be drawn in view of the health status of the animal used.

The microbiota of the animal gut is diverse and complex. It is also similar to that of humans and may help us to understand their composition, interaction and mechanism within the host. The gut microbiota is said to be a crucial part of the host performing several physiological, biochemical and metabolic functions. Recently, changes in the gut microbiota has been linked to several diseases such as obesity, diabetes, Alzheimer's, inflammatory bowel diseases etc. Diet has a substantial effect on the composition of gut microbiota [1]. Changes in humanized gnotobiotic mice was observed when they were switched from a low fat diet to high fat and sugar diet showing gut microbiota changes as well as differences in metabolic pathways. Bacteria of the phylum Firmicutes were found to be associated with weight gain or adiposity [2]. On the other hand, striking differences in the gut microbial composition between children from rural Africa and urban Europe was observed. The African gut microbiota had abundance of Bacteroidetes bacteria specifically Prevotella and Xylanibacter which were very low or completely absent in their European counterparts [2].

Several methods have been used for identification of the microorganisms present in the gut environment. Conventional method is culturing microorganisms from fecal and intestinal samples and identifying using phenotypic identification methods. Microscopic techniques are also used to differentiate bacteria based on their shape 
and arrangement. Molecular methods like species specific PCR and real-time PCR are also being used for identification of gut microbiota.

Flow cytometry is increasingly being used in biomedical research for rapid identification of cell parameters and also provides statistical accuracy. It has wide applications in Microbiology such as counting of bacterial cells, studying the antibiotic susceptibility of clinical samples, bacterial cell cycle analysis, aquatic microbial studies etc. Flow cytometer can process thousands of cells in seconds and individually analyze them. Thus, several different characteristics of each cell can be studied using multiparameters to define sub-populations and different cell types. As measurements are taken from single cells, heterogeneity within a population can easily be detected and quantified. The data analysis software allows analysis of a huge amount of data generated from multi-parameter data acquisition. Further, the procedure allows both quantitative and qualitative analysis of samples. Heterogeneous populations in any given sample can easily be identified using flow cytometry. Therefore, the aim of the study was to evaluate the usefulness of flow cytometry for rapid detection of microbial species present in the gut of rodents.

\section{MATERIAL AND METHODS}

\section{A. Isolation and Identification of Fecal Microbiota}

Fresh samples from the gut of different treatment mice $(\mathrm{C} 57 \mathrm{Bl} / 6 \mathrm{~J})$ groups i.e. control, low fat diet (LFD) and high fat diet (HFD) were collected once a week. The experimental animals were procured from Central Animal Facility, IISc., Bangalore and the feeds were obtained from Rayans Biotechnologies Pvt. Ltd., Hyderabad. Bedding material used is corn cobb from Sagar Industries, Bangalore. One gram of the samplewas suspended in $1 \mathrm{X}$ phosphate buffer saline (PBS) and centrifuged at $800 \mathrm{rpm}$ for 2 mins to remove debris. Different aliquots of the supernatant were stored at $-20^{\circ} \mathrm{C}$ until further analysis. Then the samples $(10 \mu \mathrm{l})$ were plated on nutrient agar plates and incubated at $37^{\circ} \mathrm{C}$ for $24 \mathrm{~h}$. Individual colonies obtained were subjected to biochemical analysis

\section{B. Enumeration of Microbial Load}

Bacterial load of the gut samples were determined by serial dilution, plating and total viable count method [3]. It was assumed that each colony on the plate developed from a single cell. The bacterial load was statistically analyzed by ANOVA.

\section{Serial Dilution and Total Viable Count}

Sample (100 mg) was aseptically suspended in $4.5 \mu \mathrm{l}$ of $1 \mathrm{X}$ PBS and mixed well to obtain a homogenous suspension. The samples were then serially diluted up to $10^{-8}$ dilution and were plated on nutrient agar and incubated at $37^{\circ} \mathrm{C}$ for $24 \mathrm{~h}$. For each dilution three replicates were maintained. Colonies obtained were counted and expressed as CFU/ml.

\section{Bacterial Count Using Flow Cytometry}

Isolates obtained from the gut samples of $\mathrm{C} 57 \mathrm{Bl} / 6 \mathrm{~J}$ were grown on nutrient broth. Individual samples were diluted using PBS. Counting was done on $1^{\text {st }}, 5^{\text {th }}, 10^{\text {th }}$ and $15^{\text {th }}$ week.The samples were then serially diluted up to $10^{-8}$ dilution and used for counting on FACS (BD Biosciences, USA). The viability and dilution of the cells were kept constant during the flow cytometry studies. Sample flow rate was always maintained at "Low" allowing between 500 to 600 cells to pass the laser beam per second.

\section{RESULTS AND DISCUSSION}

To isolate and identify bacteria present in rodent gut samples from control, LFD and HFD mice were plated and several colonies were differentiated using various morphological and microbiological methods. Colony counts (Table I and Figure 1) and characters such as color, elevation, margin and surface were noted. The results show that the number of bacterial colonieswere more in the case of control and LFD, while in HFD there was a significant decrease. Also, the number of bacterial colonies decreased with increase in the number of days and on the $15^{\text {th }}$ week, none of the colonies were seen when fed with HFD. But in control and LFD, the number of colonies was maintained in the same level.

TABLE I COLONY COUNTS OF BACTERIA FROM DIFFERENT GROUPS BY PlATE METHOD

\begin{tabular}{|c|c|c|c|}
\hline Weeks & Control & LFD & HFD \\
\hline $1^{\text {st }}$ Week & $>500$ & $\sim 350$ & $\sim 200$ \\
\hline $5^{\text {th }}$ Week & $>500$ & $>500$ & $\sim 100$ \\
\hline $10^{\text {th }}$ Week & $>500$ & $\sim 400$ & NIL \\
\hline $15^{\text {th }}$ Week & $>400$ & $\sim 300$ & NIL \\
\hline
\end{tabular}
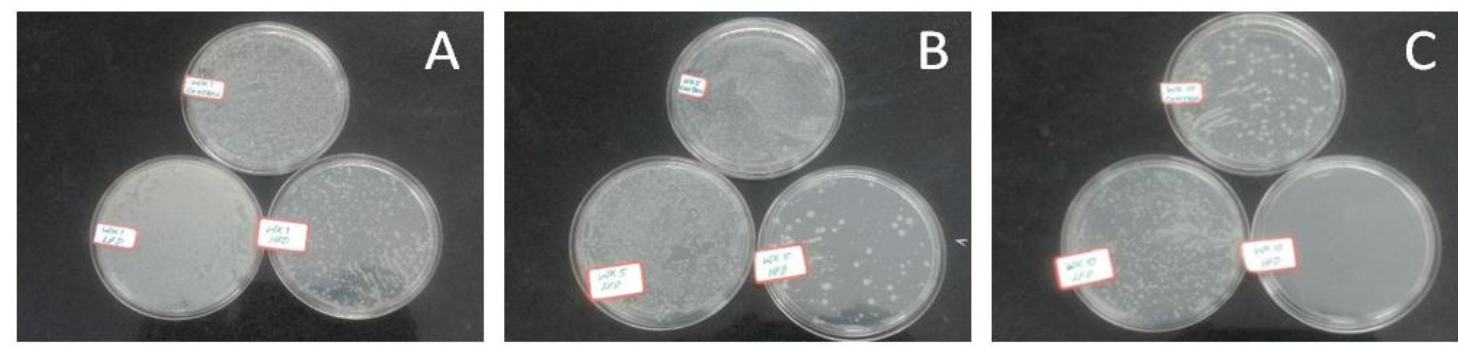

Fig. 1 Representative plate showing colonies obtained from different groups of mice A- Control, B - LFD and C - HFD 
The number of colonies was also counted using flow cytometer which showed reduced number of cells in HFD fed groups compared to control and LFD groups. The number of colonies in HFD fed mice was lesser in number. The total number bacterial counts were 61329,38411 and 5230 for control, LFD and HFD, respectively (Figure 2).

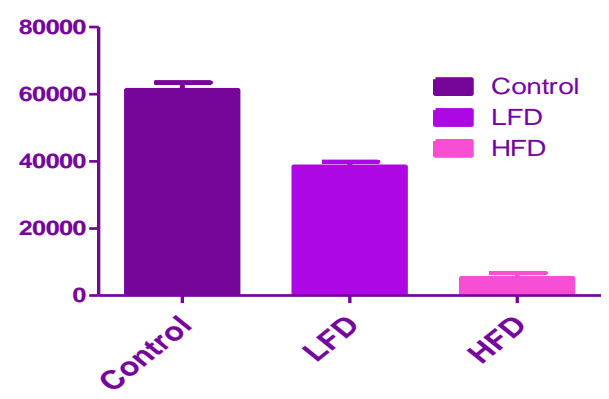

Fig. 2 Bacterial Count from Gut Samples of Control, LFD and HFD by Flow Cytometry

Diet is said to have a major influence on the gut microbial composition. Diet induced changes in the microbiota have been linked to impaired intestinal function $[1,2]$. Fecal pellets of high fat and low fat diet fed mice were assessed for microbial load by conventional and flow cytometric method. The microbial numbers were found to be low in HFD fed mice $(<10 \% \%$ less) when compared to LFD and control mice. High fat diet has a significant effect on the mouse caecal microbiota that causes compositional changes to major alterations in bacterial physiology and metabolism [4]. After ingestion of food, the dietary contents pass through the colon forming fecal matter in the process. It is plausible that the differential changes of microbiota profile in the cecal and colonic mucosa may be due to the differences in the function of these intestine segments and their response to dietary components. Studies have shown differences in the quantity and proportion of dominant phyla namely Bacteroidetes and Firmicutes. Ley et al., [5] (2014) showed that obesity can be associated with altered gut microbiota. It has been observed that HF feeding alters the diversity and composition of intestinal microbiota. Some of the studies have revealed the differences in the quantity and proportion of two dominant divisions of gut bacteria namely Bacteriodetes and Firmicutes.

In summary, we demonstrated that diet can alter the biochemical composition of the gut microbiota either by shifting phylotype composition or the activity of bacterial cells. While many studies have shown that microbial diversity is altered by dietary changes [6 -9], much less is known about the impact of diet on the metabolic potential of gut microbiota [10,11]. This alteration might influence fat absorption providing mechanist insight into how microbiota diet interaction regulates host energy balance. Some studies have reported tentative links between certain species of microbes and obesity $[1,12]$. These data strongly suggested that intestinal microbiota could be responsible for changes of metabolic endotoxemia and for the onset of the corresponding diseases similar to diabetes [13].
Additionally, flow cytometry analysis was done to ascertain whether cytometers can be used in microbial species identification. Cytometers readily detects bacteria, and distinguish them from optical and electronic noise. The results suggest the usefulness of flow cytometry for rapid identification differentiation of isolates from fecal samples. Our work is supported by earlier reports in microbial identification using flow cytometry by Gant et al. [14] (1993); Davey et al. [15] (2003). Advantages of flow cytometer includes that large number of cells can be individually analyzed faster and also individual cell analysis helps in understanding heterogeneity among microbial cells.

\section{CONCLUSION}

Microbial enumeration of mice can reveal changes in gut microbiota. Flow cytometry was employed to evaluate its efficacy as a tool for rapid identification of bacteria. Flow cytometer analysis from side and forward scatter plots revealed four distinct patterns which correlated with the results obtained from conventional methods. Thus, this study shows that flow cytometry can be effectively used to detect and differentiate various microorganism based on their size and granularity. However, care should be taken to avoid data arising from variable factors.

\section{REFERENCES}

[1] P. J. Turnbaugh, V. K. Ridaura, J. J. Faith, F. E. Rey, R. Knight and J. I. Gordon, "The effect of diet on the human gut microbiome: A Metagenomic Analysis in humanized gnotobiotic mice", Science Translational Medicine, Vol. 1, No. 6, pp.6ra14-6ra14, 2009.

[2] C. De Filippo, D. Cavalieri, M. Di Paola, M. Ramazzotti, J. B. Poullet, S. Massart, S. Collini, G. Pieraccini and P. Lionetti, "Impact of diet in shaping gut microbiota revealed by a comparative study in children from Europe and rural Africa", Proceedings of the National Academy of Sciences, Vol. 107, No. 33, pp. 14691-14696, 2010.

[3] J. G. Cappuccino and N. Sherman, Microbiology: A Laboratory Manual, 1996.

[4] P. D. Cani and N. M. Delzenne, "Gut microflora as a target for energy and metabolic homeostasis", Current Opinion in Clinical Nutrition and Metabolic Care, Vol. 10, No. 6, pp. 729-734, 2007.

[5] S. H. Ley, O. Hamdy, V. Mohan and F. B. Hu, "Prevention and management of type 2 diabetes: dietary components and nutritional strategies", The Lancet, Vol. 383, No. 9933, pp. 1999-2007, 2014.

[6] T. Clavel, M. Fallani, P. Lepage, F. Levenez, J. Mathey, V. Rochet, M. Sérézat, M. Sutren, G. Henderson, C. Bennetau-Pelissero and F. Tondu, "Isoflavones and functional foods alter the dominant intestinal microbiota in postmenopausal women", The Journal of Nutrition, Vol. 135, No. 12, pp. 2786-2792. 2005.

[7] I. Martínez, G. Wallace, C. Zhang, R. Legge, A.K. Benson, T. P Carr, E. N. Moriyama and J. Walter, "Diet-induced metabolic improvements in a hamster model of hypercholesterolemia are strongly linked to alterations of the gut microbiota", Applied and Environmental Microbiology, Vol. 75, No. 12, pp. 4175-4184, 2009.

[8] R. Jumpertz, D.S. Le, P.J. Turnbaugh, C. Trinidad, C. Bogardus, J.I. Gordon and J. Krakoff, "Energy-balance studies reveal associations between gut microbes, caloric load, and nutrient absorption in humans", The American Journal of Clinical Nutrition, Vol. 94, No. 1, pp. 58-65, 2011

[9] A. L. Kau, P. P. Ahern, N. W. Griffin, A. L. Goodman and J. I. Gordon, "Human nutrition, the gut microbiome and the immune system", Nature, Vol. 474, No. 7351, p. 327, 2011.

[10] R. Martin, A. Nauta, K. Ben Amor, L. Knippels, J. Knol and J. Garssen, "Early life: gut microbiota and immune development in infancy", Beneficial Microbes, Vol. 1 No. 4, pp. 367-382, 2010. 
[11] B. D. Muegge, J. Kuczynski, D. Knights, J. C. Clemente, A. González, L. Fontana, B. Henrissat, R. Knight and J. I. Gordon, "Diet drives convergence in gut microbiome functions across mammalian phylogeny and within humans", Science, Vol. 332, No. 6032, pp. 970-974, 2011.

[12] M. Million, M. Maraninchi, M. Henry, F. Armougom, H. Richet, P. Carrieri, R. Valero, D. Raccah, B. Vialettes and D. Raoult, "Obesityassociated gut microbiota is enriched in Lactobacillus reuteri and depleted in Bifidobacterium animalis and Methano brevi bactersmithii", International Journal of Obesity, Vol. 36, No. 6, p. $817,2012$.
[13] P. D. Cani, B. Rodrigo, C. Knauf, A. Waget, A. M. Neyrinck, N. M Delzenne and R. Burcelin, "Changes in gut microbiota control metabolic endotoxemia-induced inflammation in high-fat dietinduced obesity and diabetes in mice", Diabetes, 2008.

[14] V. A. Gant, G. Warnes, I. Phillips and G. F. Savidge, "The application of flow cytometry to the study of bacterial responses to antibiotics", Journal of Medical Microbiology, Vol. 39, No .2, pp. 147-154, 1993

[15] H. M. Davey, "Flow cytometric techniques for the detection of microorganisms", In Advanced Flow Cytometry: Applications in Biological Research, Springer, Dordrecht, pp. 91-97, 2003. 